\title{
Preclinical evaluation of patient-derived cells shows promise for Parkinson's disease
}

\author{
Jun Takahashi \\ Department of Clinical Application, Center for iPS Cell Research and Application, Kyoto University, Kyoto, Japan.
}

\begin{abstract}
Parkinson's disease (PD) is a neurodegenerative disease caused by the progressive loss of dopaminergic (DA) neurons in the midbrain projecting to the striatum, which leads to motor dysfunctions, such as bradykinesia (slowed movement), rigidity, and tremors. To replace the lost cells, the transplantation of DA neurons derived from embryonic stem cells or induced pluripotent stem cells (iPSCs) has been considered. In this issue of the $J C I$, Song et al. report on their development of an iPSC induction and differentiation protocol that can promote the realization of autologous transplantation to treat PD patients with their own cells.
\end{abstract}

\section{Cell transplantation for Parkinson's disease}

Parkinson's disease (PD) is a neurodegenerative disease in which the number of dopaminergic (DA) neurons that project from the midbrain substantia nigra to the striatum progressively decrease. As a result, patients mainly exhibit motor dysfunctions, such as bradykinesia (slowed movement), rigidity, and tremors. To replace the lost DA neurons, the transplantation of fetal midbrain, which contains DA neurons, has been attempted since 1987. A number of clinical cases have proved that this treatment can improve patient symptoms (1). Moreover, positive effects for over 10 years have been reported (2). Now, a new clinical trial is ongoing in Europe that seeks to optimize the treatment protocols of this strategy (3). However, there are several issues regarding fetal-based treatment, including ethical concerns using fetal tissue, difficulty in obtaining a sufficient amount of fetal brain tissue, and contamination of serotonergic neurons, which may cause dyskinesia (involuntary movement). Although the efficacy of fetal cell transplantation has been demonstrated, it is not yet admitted as a standard treatment.
Embryonic stem cells (ESCs) and induced pluripotent stem cells (iPSCs) are considered alternative cell sources for DA neurons because both can differentiate into somatic cells of every organ. ESCs are derived from the inner cell mass of a blastocyst, which defines the early stage preimplantation embryo (4). On the other hand, iPSCs are induced from somatic cells, such as skin fibroblasts or blood cells, by introducing reprogramming factors (5). As a result, iPSCs result in fewer ethical problems because they avoid embryo destruction and can potentially allow autologous transplantation by using the patient's own cells, in which case there is no need for immunosuppression. To take advantage of this, the team led by Kim developed an iPSC induction and differentiation protocol to treat PD patients by autologous transplantation (6).

\section{Improving methods to generate DA neurons from iPSCs}

The process of autologous transplantation can be divided into two parts. One part establishes the iPSC line and the corre-

Related Article: p. 904

Conflict of interest: JT receives research support from Sumitomo Dainippon Pharma.

Copyright: @ 2020, American Society for Clinical Investigation.

Reference information: J Clin Invest. 2020;130(2):601-603. https://doi.org/10.1172/JCI134031. 
controlled by bone morphogenic protein (BMP), TGF/activin/nodal and Wnt signals $(6,13)$. In order to induce neural cells, it is important to inhibit both BMP and TGF/activin/nodal signals. Because this method inhibits the SMAD1/5/8 and SMAD2/3 intracellular pathways, it is called dual SMAD inhibition (10). In addition to the above, to generate DA neurons, the ventral midbrain is induced by moderately activating Wnt signals and ventralizing with Sonic hedgehog (Shh) (11, $12,14)$. The combination of these conditions results in a highly efficient method to induce DA neurons, but the possibility of residual undifferentiated cells with neoplastic potential remains $(15,16)$. One possible solution to avoid this risk is the removal of unwanted cells by sorting DA neurons with antibodies against CORIN (17), a marker for floor plate, or ALCAM (18), a marker for vascular endothelial cells in the central nervous system. Song et al. took another path. Based on the previous findings that BIRC5 (encoding survivin) is highly expressed in human ESCs/iPSCs compared with somatic cells (19), they hypothesized that the chemically inhibiting survivin would selectively eliminate any remaining undifferentiated iPSCs. Among survivin inhibitors, they chose the flavonoid quercetin and found that it eliminated undifferentiated iPSCs with greater than $99.99 \%$ efficiency (6).

\section{Future directions}

One of the merits of autologous transplantation relates to the immune response by the host brain. Previous studies have confirmed that there is only a minimum immune response to the autologous transplantation of iPSC-derived neural cells in nonhuman primate brains (20). Therefore, there is no need for immunosuppressant drugs upon transplantation, which avoids adverse effects such as liver or kidney dysfunction and a compromised host. In addition, there is minimal risk of transmitting pathogens from donor tissue. However, autologous transplantation requires establishing iPSCs, inducing DA neurons, and evaluating the induced cells for each patient. At the moment, these processes are costly, laborious, and time-consuming. Moreover, it remains unclear whether patient-derived DA neurons are appropriate for treatment, especially for familial patients in which genetic mutations exist in the cells derived for transplantation.

Although details remain unknown, the new protocol presented by Song et al. will contribute to reducing the cost and time of generating DA neurons from somatic cells (6). Especially in autologous transplantations, the efficiency of obtaining cells of good quality is critical. According to the current study, the authors analyzed five independent iPSC lines derived from a sporadic PD patient by karyotyping, quantitative real-time PCR (qRT-PCR), and whole-exome sequencing and found that four clones out of five were free of integrated plasmid DNAs and contained no somatic mutations causally implicated in cancer. Considering that the establishment of iPSC lines and these genomic evaluations are time- and cost-consuming, this efficiency is remarkable. At the same time, however, the authors made no statement about the genomic stability during differentiation. It is possible that mutations may occur during differentiation, which could prohibit the transplantation. Therefore, quality checks will be needed for the final product, not only to evaluate genomic integrity, but also to check for the contamination of animal-derived proteins, sterility, and the presence of mycoplasma or endotoxins. There is still debate about the interpretation of the genomic analysis results, in part because iPSCs are a new cell type and not all genetic mutations cause malignant phenotypes. Future genomic analyses and clinical data will help to establish quality check criteria.

Finally, Song et al. performed in vivo studies to confirm the efficacy of their DA neurons induced from sporadic PD patient-derived iPSCs and found that the cells improved the behaviors of 6-OHDAlesioned rats (6). Previously, Kikuchi et al. reported that DA neurons induced from sporadic PD patient-derived iPSCs improved the behaviors of MPTP-treated nonhuman primates (21). Although there are several reports that iPSCs derived from familial PD patients cannot give rise to healthy DA neurons, it seems that DA neurons derived from sporadic PD patients can function in the brain. Nevertheless, there is concern that PD patient-derived DA neurons might be more vulnerable to the pathology of PD than neurons from healthy individuals. In the cases of fetal cell transplantation to PD patients, an accumulation of $\alpha$-synuclein in the grafted cells was reported (2). The same observations should be made for iPSC studies, especially in the cases of autologous transplantation. Therefore, before any firm conclusions can be made about autologous treatments of PD, clinical observation over a long time period is needed.

\section{Acknowledgments}

I thank Peter Karagiannis (CiRA) for critical reading of the manuscript. Our work has been supported by the following grants: the Highway Project for Realization of Regenerative Medicine from the Ministry of Education, Culture, Sports, Science and Technology (MEXT) and the Research Center Network for Realization of Regenerative Medicine from the Japan Agency for Medical Research and Development (AMED).

Address correspondence to: Jun Takahashi, Department of Clinical Application, Center for iPS Cell Research and Application, Kyoto University, 53 Shogoin kawaharacho, Sakyo-ku, Kyoto 606-8507, Japan. Phone: 81.75.366.7052; Email: jbtaka@ cira.kyoto-u.ac.jp.

1. Barker RA, Drouin-Ouellet J, Parmar M. Cellbased therapies for Parkinson disease-past insights and future potential. Nat Rev Neurol. 2015;11(9):492-503.

2. Li W, et al. Extensive graft-derived dopaminergic innervation is maintained 24 years after transplantation in the degenerating parkinsonian brain. Proc Natl Acad Sci USA. 2016;113(23):6544-6549.

3. Barker RA, TRANSEURO Consortium. Designing stem-cell-based dopamine cell replacement trials for Parkinson's disease. Nat Med. 2019;25(7):1045-1053.

4. Thomson JA, et al. Embryonic stem cell lines derived from human blastocysts. Science. 1998;282(5391):1145-1147.

5. Takahashi K, et al. Induction of pluripotent stem cells from adult human fibroblasts by defined factors. Cell. 2007;131(5):861-872.

6. Song B, et al. Human autologous iPSC-derived dopaminergic progenitors restore motor function in Parkinson's disease models. J Clin Invest. 2020;120(2):904-920.

7. Takahashi K, Yamanaka S. Induction of pluripotent stem cells from mouse embryonic and adult fibroblast cultures by defined factors. Cell. 2006;126(4):663-676.

8. Nakagawa M, Takizawa N, Narita M, Ichisaka T, Yamanaka S. Promotion of direct reprogramming by transformation-deficient Myc. Proc Natl Acad Sci USA. 2010;107(32):14152-14157.

9. Okita K, et al. A more efficient method to gener- 
ate integration-free human iPS cells. Nat Methods. 2011;8(5):409-412.

10. Chambers SM, Fasano CA, Papapetrou EP, Tomishima M, Sadelain M, Studer L. Highly efficient neural conversion of human ES and iPS cells by dual inhibition of SMAD signaling. Nat Biotechnol. 2009;27(3):275-280.

11. Kirkeby A, et al. Generation of regionally specified neural progenitors and functional neurons from human embryonic stem cells under defined conditions. Cell Rep. 2012;1(6):703-714.

12. Xi J, Liu Y, Liu H, Chen H, Emborg ME, Zhang SC. Specification of midbrain dopamine neurons from primate pluripotent stem cells. Stem Cells. 2012;30(8):1655-1663.

13. Murry CE, Keller G. Differentiation of embryonic stem cells to clinically relevant populations: lessons from embryonic development. Cell.
2008;132(4):661-680.

14. Kriks S, et al. Dopamine neurons derived from human ES cells efficiently engraft in animal models of Parkinson's disease. Nature. 2011;480(7378):547-551.

15. Doi D, et al. Prolonged maturation culture favors a reduction in the tumorigenicity and the dopaminergic function of human ESC-derived neural cells in a primate model of Parkinson's disease. Stem Cells. 2012;30(5):935-945.

16. Elkabetz Y, Panagiotakos G, Al Shamy G, Socci ND, Tabar V, Studer L. Human ES cell-derived neural rosettes reveal a functionally distinct early neural stem cell stage. Genes Dev. 2008;22(2):152-165.

17. Doi D, et al. Isolation of human induced pluripotent stem cell-derived dopaminergic progenitors by cell sorting for successful transplantation. Stem Cell Reports. 2014;2(3):337-350.
18. Bye CR, Jönsson ME, Björklund A, Parish CL, Thompson LH. Transcriptome analysis reveals transmembrane targets on transplantable midbrain dopamine progenitors. Proc Natl Acad Sci USA. 2015;112(15):E1946-E1955.

19. Lee MO, et al. Inhibition of pluripotent stem cell-derived teratoma formation by small molecules. Proc Natl Acad Sci USA. 2013;110(35):E3281-E3290.

20. Morizane A, et al. Direct comparison of autologous and allogeneic transplantation of iPSC-derived neural cells in the brain of a non-human primate. Stem Cell Reports. 2013;1(4):283-292.

21. Kikuchi T, et al. Human iPS cell-derived dopaminergic neurons function in a primate Parkinson's disease model. Nature. 2017;548(7669):592-596. 